\title{
Assessment of the Bond Strength and Mode of Failure of Glass Fibre Posts Luted using Two Different Adhesives: An In Vitro Analysis
}

\author{
${ }^{1}$ Iswariya Rajan, ${ }^{2}$ Vaiyapuri Ravi, ${ }^{3}$ Arthanarieswaran A Sivakumar, ${ }^{4}$ Jambai SK Sivakumar, \\ ${ }^{5}$ Anjaneya S Prasad, ${ }^{6}$ Saravanapriyan Soundappan
}

\begin{abstract}
Aim: The aim of this study is to assess the bond strength and the mode of failure of a glass fibre-post luted using two different adhesives.
\end{abstract}

Materials and methods: Fourty human permanent maxillary central incisors were decoronated, endodontically treated, divided into two groups $(n=20)$, after post space preparation; group I: clear post RO (Dispodent) and Variolink II (Ivoclar Vivadent); group II: clear post RO (Dispodent) and Multilink N(Ivoclar Vivadent). The roots were sectioned to a thickness of $2 \pm 0.5$. Universal testing machine was used for testing the push-out bond strength. By dividing bond value recorded in Newton $(\mathrm{N})$ and the area of bonded interface, the bond strength was derived in megapascal (MPa). After evaluating the push-out strength, the samples were analysed using a stereomicroscope to check the mode of failure.

Results: Group I showed significantly higher push-out bond strength than Group II. Adhesive bond failure between dentin and luting cement and post and was most commonly observed

Conclusion: Group I utilising Variolink II as luting cement which employs total etch technique had higher mean push-out bond strength. Bond failure between cement-dentin interface followed by post cement interface was observed as the most common type of failure, impling difficulty in a post cement dentin interface bond.

Clinical significance: The success of endodontic treatment depends not only on elimination of pulpal/periradicular pathology but also on post-endodontic management involving the maintenance of extensively damaged tooth in a functional state. In an attempt to prevent the occurance of catastrophic events like vertical root fracture associated with metal posts, fibre reinforced glass posts were introduced, which mimic dentin in relation to elastic modulus. In addition to this, improvements made in adhesive techniques further led to the popularity of these fibre reinforced posts.

Keywords: Adhesion, Bond failure, Endodontically treated teeth, Esthetic rehabilitation, Fiber posts modules of elasticity, Glass fibre post, Mutilated teeth, Post and core, Post retained crowns, Push-out test, Resin cements.

How to cite this article: Rajan I, Ravi V, Sivakumar AA, Sivakumar JSK, Prasad AS, Soundappan S. Assessment of the

\footnotetext{
${ }^{1}$ Postgraduate Student, ${ }^{2}$ Professor and HOD, ${ }^{3}$ Professor, ${ }^{4-6}$ Reader

${ }^{1-6}$ Vivekanandha Dental College for Women, Eleyampalayam, Tiruchengode, Tamil Nadu, India
}

Corresponding Author: Vaiyapuri Ravi, Professor and HOD, Vivekanandha Dental College for Women, Eleyampalayam, Tiruchengode, Tamil Nadu, India, e-mail: vai.ravi@gmail.com
Bond Strength and Mode of Failure of Glass Fibre Posts Luted using Two Different Adhesives: An In Vitro Analysis. J Oper Dent Endod 2018;3(2):57-62

Source of support: Nil

Conflict of interest: None

\section{INTRODUCTION}

Combination of endodontic and prosthetic treatments play an important role in the maintenance of extensively damaged tooth in a functional state. ${ }^{1}$ Traditionally, posts made of metal alloys were used in such cases that resulted in a complex combination of physical properties between metal and the dentin. In a clinical situation, this combination may predispose the offending tooth to vertical fracture thus affecting the prognosis of the resultant restoration. ${ }^{2}$ Attempting to prevent the occurrence of such catastrophic failure, fiber-reinforced glass posts were introduced, which have an elastic modulus similar to that of dentin. ${ }^{3}$ In addition to this, improvements made in adhesive techniques further led to the popularity of these fiber-posts. In the literature review, the performance of adhesively bonded family research council (FRC) posts has been reported to have positive results in both prospective and retrospective studies. Although studies have reported catastrophic root fracture to become a rare occurrence when using glass-fiber posts, adhesive failure (clinically seen as de-bonding of post and crown) remain to be a major clinical complication. ${ }^{4}$ The most common failure with the use of fiber-reinforced composite posts is debonding, as the bonding capacity is influenced by a number of factors such as hydration of root dentin, use of surface conditioning agents, type of adhesive material used, differences in density and orientation of dentinal tubules. ${ }^{5}$ Hence these factors should be addressed to achieve a good bond between post-cementdentin interface. The objective of this study was to evaluate the push-out bond strength of a glass fiber post luted using two different types of resin cements and to assess the mode of failure (adhesive/cohesive/combination) across the interface using a stereomicroscope.

\section{MATERIALS AND METHODS}

Pulp tissues from the samples were extirpated using standardized barbed broaches (Flow Chart 1). The specimens 
Flow Chart 1: Methodology

Sample selection

(80 extracted human maxillary central incisors)

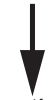

Sectioning of tooth samples at the level of cEJ using diamond discs

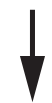

Preparation and obturation of root canals

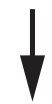

Random separation of samples into two groups $(n=20)$

Post space preparation and cementation of post

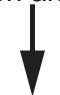

Assessment of push-out bond strength using universa testing machine and mode of bond failure under a stereomicroscope

were instrumented to a length of $0.5 \mathrm{~mm}$ short of the apex using K-files (15-40, 45-80, Mani, Japan) up to ISO size 40 as a master apical file. Step back preparation of the root canals were done and root canal conditioning was done with $17 \%$ ethylenediaminetetraacetic acid (EDTA) (Glyde file Prep root canal conditioner, Dentsply-Mailifer, China). Three percent sodium hypochlorite (Venson's India Pvt Ltd) was used for irrigation of root canals and finally irrigated with distilled water and dried using paper points. Sectional obturation of apical $4 \mathrm{~mm}$ of the root canals was done using 40 sizes gutta-percha (Dentsply-Mailifer, China), pluggers and calcium hydroxide-based sealer (Sealapex-sybron Endo) was used as the root canal sealer

After obturation, the specimens were divided into two groups randomly with each having 20 specimens. Post space was prepared with the drills provided by the manufacturer and following their usage instructions [drill size 0, 1, 2-HI-Rem (Prime dental), Clear Post (dispodent)]. The post space created was flushed with distilled water and dried with paper points to avoid over-drying of dentin.

Specimen sampling was done by randomly dividing them into two groups of 20 each as follows (Table 1).

In both the groups, the silane coupling agent was used to silanate the post space(Ivoclar Vivadent). The post space was treated with 17\% EDTA and irrigated with distilled water and dried with paper points. The post space was conditioned with 17\% EDTA, irrigated

Table 1: Grouping of samples

\begin{tabular}{llll}
\hline Sample & Sample size & Post system & Luting agent \\
\hline Group I & 20 & Clear Post & Variolink LI \\
Group II & 20 & Clear Post & Multilink N \\
\hline
\end{tabular}

with distilled water and dried with paper points. In samples of Group I where the luting agent used was Variolink II, the prepared post space was etched with $37 \%$ orthophosphoric acid for $15 \mathrm{~s}$ and rinsed and dried with paper points. The post space was coated with a Scotchbond (3M ESPE), and after 10 seconds the excess was removed with paper points. Samples were polymerized using a light curing unit (LED) for 20 seconds with the tip directed at the entrance of the post space parallel to the long axis. Luting agent was mixed as guided by the manufacturer and placed using paste carriers into the prepared post space and the prefabricated post was luted to the full depth of the canal under digital pressure and the excess was immediately removed with the help of a probe. Storage of the samples was done at room temperature in distilled water.

\section{Push-out Tests}

Sectioning of the samples was done perpendicular to the long axis at the coronal third with diamond discs, to obtain slices of $2 \pm 0.05 \mathrm{~mm}$ thickness (Figs 1 and 2). The thickness of the sectioned samples and radius of the

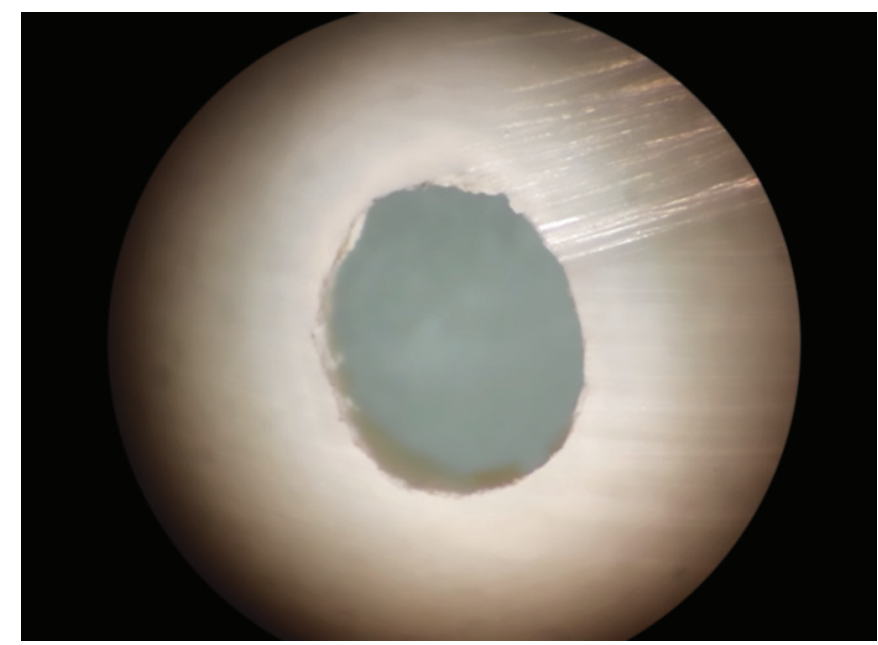

Fig. 1: Type A (Adhesive failure post-cement interface)

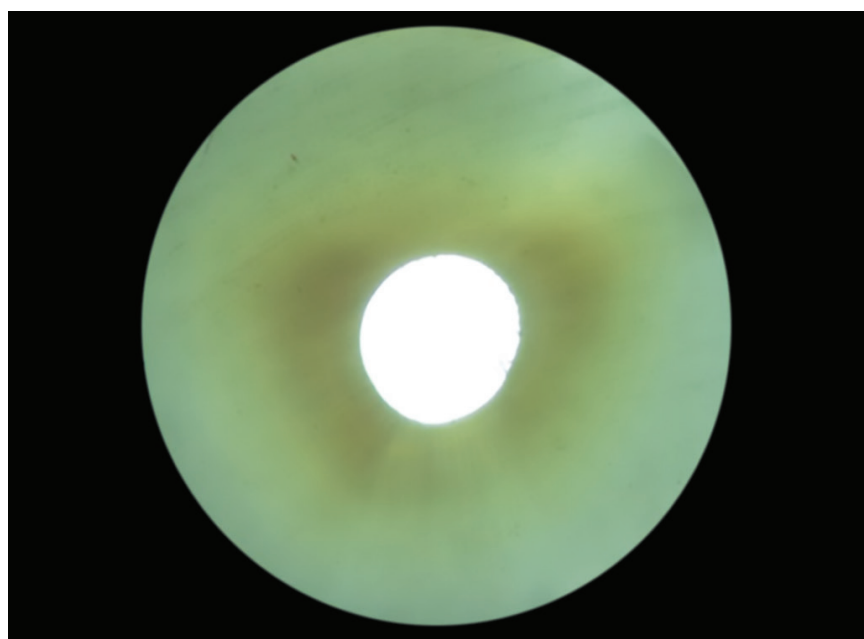

Fig. 2: Type B (Adhesive failure cement-dentin interface) 
canals were measured with Vernier Calliper. Push-out tests were performed using a universal testing machine (Lloyd Instruments, UK) with a crosshead speed of $1 \mathrm{~mm}$ per minute with the push-out jig being attached to the testing machine. The push-out pin of diameter $1 \mathrm{~mm}$ was used. The pin was centered on the post space without causing stress on the post space walls. Force was centered from the apical side so that resistance to the post displacement can be avoided as post space taper will contribute to the movement of the post system. The value of force at which bond failure occurs was recorded in Newton $(\mathrm{N})$, and the ratio of the recorded value with the area $\left(\mathrm{mm}^{2}\right)$ of the bonded interface was obtained to express the bond strength in MPa. The area of bonded surface each specimen was calculated using the formula, $\mathrm{A}=2$ Пrh, wherein,

$\Pi$ being constant at 3.14

$r$ being half the diameter of the bonded surface of the post

$h$ being the thickness of the specimen (2 mm-constant)

Debonding Stress $(\mathrm{MPa})=\frac{\text { Debonding force }(\mathrm{N})}{\text { Area }\left(\mathrm{mm}^{2}\right)}$

\section{Statistical Analysis}

The obtained data were analyzed using the one-sample Kolmogorov-Smirnov test using Statistical Package for the Social Sciences (SPSS) software version 10.0 to assess the significance of group statistics with $\mathrm{p}<0.01(99 \%$ significance), (Table 2).

\section{Stereo Microscope Analysis}

The samples were analyzed to assess the type of failure using stereomicroscope at the original magnification of

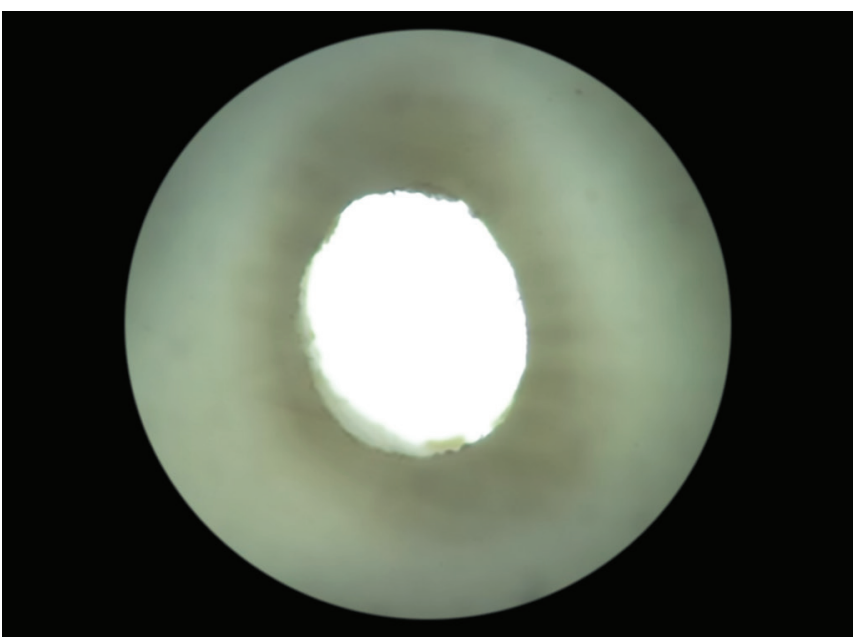

Fig. 3: Type E (Combination of the above)

40X. Based on the observations the mode of failures was classified as,

- Type A: Adhesive failure between post and luting material (Fig. 1)

- Type B: Adhesive failure between dentin and luting material (Fig. 2)

- Type C: Cohesive failure of post system (Fig. 3)

- Type D: Cohesive failure of luting material

- Type E: Mixed type; combination of any two of the above-mentioned types.

\section{RESULTS}

Group I samples where Variolink II was used to lute the clear post, showed higher mean bond strength readings compared to group II where Multilink N was used, ( $p<0.001$ statistically significant difference), (Table 3 ). Type B ( failure at the cement-dentin interface) was the most common mode of failure, (Table 4).

Table 2: Statistical analysis

\begin{tabular}{lllll}
\hline & \multicolumn{3}{c}{ One-sample Kolmogorov-Smirnov test } \\
\cline { 2 - 5 } Group & & Area $\left(\mathrm{mm}^{2}\right)$ & Deboding force $(\mathrm{N})$ & $\begin{array}{l}\text { Compressive strength of } \\
\text { post systems (Mpa) }\end{array}$ \\
\hline \multirow{2}{*}{ Group I } & Kolmogorov-Smirnov Z & 0.93 & 0.77 & 0.68 \\
& $\mathrm{P}$ & 0.35 & 0.59 & 0.74 \\
\hline \multirow{2}{*}{ Group II } & Kolmogorov-Smirnov Z & 0.87 & 0.78 & 0.44 \\
& $\mathrm{P}$ & 0.44 & 0.58 & 0.99 \\
\hline
\end{tabular}

Table 3: Statistical analysis

\begin{tabular}{|c|c|c|c|c|c|c|}
\hline & Group & $N$ & Mean & $S D$ & $t$ & $p$ \\
\hline \multirow[t]{2}{*}{ Area of bonded interface $\left(\mathrm{mm}^{2}\right)$} & Group I & 20 & 16.89 & 1.26 & 0.150 & 0.882 \\
\hline & Group II & 20 & 16.83 & 1.38 & & \\
\hline \multirow[t]{2}{*}{ Debonding force $(\mathrm{N})$} & Group I & 20 & 134.86 & 54.77 & 8.619 & $<0.001^{* *}$ \\
\hline & Group II & 20 & 28.09 & 8.28 & & \\
\hline \multirow[t]{2}{*}{ Compressive strength $\mathrm{Mpa}$} & Group I & 20 & 7.88 & 3.61 & 7.657 & $<0.001^{* *}$ \\
\hline & Group II & 20 & 1.66 & 0.44 & & \\
\hline
\end{tabular}


Table 4: Mode of failure

\begin{tabular}{llllll}
\hline Group & Type A & Type B & Type C & Type D & Type E \\
\hline I & 5 & 15 & - & - & - \\
II & 7 & 11 & - & - & 2 \\
\hline
\end{tabular}

Type A-Adhesive failure at cement-dentin interface

Type B-Adhesive failure at post-dentin interface

Type E-Combination of cohesive and adhesive type of failure

- Independent Samples t-test. The Independent samples t-test compares the means of two independent groups in order to determine whether there is statistical evidence that the associated population means are significantly different. The Independent Samples t-test is a parametric test.

- In statistics, the Mann-Whitney U-test (also called the Mann-Whitney-Wilcoxon (MWW), Wilcoxon rank-sum test, or Wilcoxon-Mann-Whitney test) is a nonparametric test of the null hypothesis that it is equally likely that a randomly selected value from one sample will be less than or greater than a randomly selected value from a second sample.

Unlike the t-test it does not require the assumption of normal distributions. It is nearly as efficient as the t-test on normal distributions.

This test can be used to determine whether two independent samples were selected from populations having the same distribution; a similar nonparametric test used on dependent samples is the Wilcoxon signed-rank test.

- In statistics, the Kolmogorov-Smirnov test (K-S test or KS test) is a nonparametric test of the equality of continuous, one-dimensional probability distributions that can be used to compare a sample with a reference probability distribution (one-sample K-S test), or to compare two samples (twosample K-S test). It is named after Andrey Kolmogorov and Nikolai Smirnov.

The Kolmogorov-Smirnov statistic quantifies a distance between the empirical distribution function of the sample and the cumulative distribution function of the reference distribution, or between the empirical distribution functions of two samples. The null distribution of this statistic is calculated under the null hypothesis that the sample is drawn from the reference distribution (in the one-sample case) or that the samples are drawn from the same distribution (in the two-sample case). In each case, the distributions considered under the null hypothesis are continuous distributions but are otherwise unrestricted.

\section{DISCUSSION}

Adhesive techniques have been a boon to restorative dentistry as they enable the preservation of maximal amount of sound tooth structure. This has further expanded the bonding of posts to root dentin. Use of fiber posts minimizes the removal of root dentin, thus preserving the root structure, enhancing the long-term survival of post-endodontic restoration. Glass-fiber posts being translucent gives better esthetic properties to the resultant restoration. ${ }^{2}$ Using prefabricated posts, minimizes the chair side working time and simplifies the procedure, thus the procedure can be completed in a single visit. Bond failure due to dislodgment of the post within the root canal can be due to the stress on the adhesive cement layer when exposed to functional loading. Factors such as the degree of hydration of radicular dentin, use of surface conditioning agent, type of adhesive used, cavity configuration factor (C-factor), nature of root canal sealer used, the differences in the density and orientation of the dentinal tubules. ${ }^{6}$ Adhesive cements can bind both to dentin and the post used. However, the high configuration factor within the root canal system disrupts the cement-dentin interface, thereby causing gaps which impairs the bonding efficacy of the adhesive cement used. The dislocation resistance of fiber post bonded using adhesive cement is mainly derived from sliding friction rather than real adhesion to the radicular dentin. ${ }^{7}$ When resin cements associated with the adhesive system are used, the incompatibility between the adhesive and the resin can, in turn, lead to compromised luting ability. This increases the risk of postoperative failure. To overcome this disadvantage, manufacturers started to develop simpler luting materials which are self-adhesive. Thus they don't require any pre-treatment procedure of the dental surface to be bonded. ${ }^{8}$ Hence, such cements could be used for luting of posts in a single step. Besides the ease of use, to obtain stable bonding and adequate mechanical properties, proper polymerization of cement is essential. For the initiation of the polymerization reaction, a light source is required when photo-polymerizable resin cements are used for post cementation. If the energy from the light source is not sufficient enough to promote polymerization reaction, it would eventually lead to post failure due to the lack of adhesion between the post and the root dentin. ${ }^{9}$ Clear posts of variable sizes 0.70 , 0.90, 1.10 which are made of glass fibers were used in this study. These posts are translucent and radiopaque thereby enhancing the polymerization reaction of the resin luting cement being used. In this study both the luting cements used were a dual cure in which Variolink II uses a separate adhesive system whereas Multilink $\mathrm{N}$ is a self-adhesive luting cement. Literature review shows many methods to analysis the bond strength which included tensile, microtensile tests, push-out, and pull-out tests. It was in the 1970s that the push-out tests were first used in dentistry. These tests were used for assessment of the bond strength of materials to root dentin and were first reported in 1996. These push-out tests are useful in providing a better estimation of bond strength values when compared with that of conventional shear tests. The results obtained from push-out tests are more reliable as the fracture happens parallel to the dentin-bonding interface thus it is a true shear test. Besides, push-out tests are comparatively superior in terms of reliability to 
that of micro-tensile bond strength tests, when it comes to post system. Such tests provide smaller adhesive areas where more uniform stress distribution occurs on the adhesive interface, only a few specimens were recorded to be lost during experimentation, with lower standard deviation values and the ease of performance of the test procedure. ${ }^{10}$ Although literature presents the use of specimens with wide variations in thickness (1-4 mm), in current study thickness of $2 \pm 0.05 \mathrm{~mm}$ is standardized which could present lower friction areas and comparatively lesser chance of over estimation of the results than when thicker slices were used. According to the inference of this study, group I which included samples of clear post luted with Variolink II showed the higher mean values. The possible reason for this significant variation in result values between the cements used could be due to the nature of adhesive procedure among the cements. Variolink II requires the use of a separate adhesive system which involves etching of the dentin surface using 37\% phosphoric acid following which application of an adhesive before the cementation of the posts needs to be done. However, the additional advantage of using an adhesive system is that the enhanced mechanism of adhesion and also the removal of thick smear layers over the surface of dentin when the surface is etched with 37\% phosphoric acid, and this enables superior penetration of the resin cement to the dentin substrate. In group II where Multilink N was used as a luting cement, the resultant bond strength values were found to be significantly less compared to the other group. The difference in Multilink $\mathrm{N}$ is that it is a dual-curing, self-etching resin cement which does not involve any surface conditioning of root dentin before luting of the post system into the prepared root canals. The self-etching primers of Multilink N could not penetrate through the thick smear layers of the dentinal walls which made adhesive infiltration difficult in samples of group II. These results were similar to the previous studies done by Kadam et al. ${ }^{1}$ when selfadhesive resin cement was compared with a resin cement which required a separate adhesive system for cementation of posts. Considering the mode of failure analyzed in this study, it could be seen that type B (failure at the dentin-cement interface) was found to be predominant. This is in accordance with previous studies conducted using similar methods. The probable reason for such an occurrence could be attributed to the effect of polymerization stress occurring at the interface between dentin and the resin cement. The root canal configuration which is responsible for high $\mathrm{C}$-factor makes the resin-cement interface opposite to the cavity walls to compete with the bond strength of the adhesive area. ${ }^{11}$ The second common mode of failure was between the adhesive interface of the post and resin cement. The adhesion is mainly physico- mechanical and lack of chemical interaction between the post and adhesive cement. Considering the influence of the resin cement thickness on push-out bond strength of glass fibre-posts, a study by Maria et al. demonstrated a strong negative correlation between the thickness of resin cement and bond strength values, thus it could be stated that the greater the thickness of the cement, the lower the bond strength and vice versa. According to this study, it should be emphasized that clinicians should seek the lowest possible thickness of the resin luting cement when using glass fibre-posts

\section{CLINICAL SIGNIFICANCE}

The success of endodontic treatment depends not only on elimination of pulpal/periradicular pathology but also on post-endodontic management involving the maintenance of extensively damaged tooth in a functional state. In an attempt to prevent the occurance of catastrophic events like vertical root fracture associated with metal posts, fibre reinforced glass posts were introduced, which mimic dentin in relation to elastic modulus. In addition to this, improvements made in adhesive techniques further led to the popularity of these fibre reinforced posts.

\section{CONCLUSION}

It can be concluded taking into account the limitations of this: The mean push-out values of group I in which clear posts were luted using Variolink II showed higher values compared to group II samples in which Multilink $\mathrm{N}$ was used. Among the samples, adhesive failure at the cement-dentin interface was the most commonly observed failure mode, followed by failure at the postcement interface.

\section{REFERENCES}

1. Kadam A, Pujar M, Patil C. Evaluation of push-out bond strength of two fiber-reinforced composite posts systems using two luting cements in vitro'. Journal of Conservative Dentistry 2013;16(5):444-448.

2. Cheung $W$. A review of the management of endodontically treated teeth. JADA 2005;136(5):611-619.

3. Bateman G, Ricketts DNJ, Saunders WP. Fiber-based post systems: a review. British dental journal 2003;195(1):43-48.

4. Pereira JR, Valle AL, Ghizoni JS, Só MV, Ramos MB, Lorenzoni FC. Evaluation of push out bond strength of four luting agents and SEM observation of the dentine/fibreglass bond interface. International endodontic journal. 2013;46(10):982-992.

5. Chadha R, Taneja S, Kumar M, Gupta S. An in-vitro comparative evaluation of depth of tubular penetration of three resin based root canal sealers. Journal of conservative dentistry 2017;15(1):18-21.

6. Montagner AF, Carvalho MPM, Susin A. 'Microshear bonding effectiveness of different dentin regions', Indian journal of dental research 2013;26(2):pp.131-135. 
7. Giachetti L, Scaminaci Russo D, Baldini M, Bertini F, Steier L, Ferrari M. Push-out strength of translucent fibre posts cemented using a dual-curing technique or a light-curing self-adhering material. International endodontic journal. 2012;45(3):249-256.

8. Mumcu E, Erdemir U, Topcu FT. Comparison of micro pushout bond strength of two fiber posts luted using simplified adhesive approach. Dental materials journal 2010;29(3): 199-218.
9. Le Bell AM, Lassila VJ, Kangasniemi I, Vallitu PK. Bonding of fibre-reinforced composite post to root canal dentin. Journal of Dentistry 2005;33:533-539.

10. Mazzitelli C, Monticelli F. Evaluation of the push out bond strength of self-adhesive resin cements to fibre posts. International dentistry 2008;11(6):54-60

11. Tay F.R, Loushine RJ, Lambrechts P, Weller RN, Pashley DH. Geometric factors affecting dentin bonding in root canals: A theoretical modeling approach. Journal of endodontics 2005;31(8):584-589. 